\title{
ON EQUIVARIANT DEFORMATION OF MAPS
}

\author{
ANTONIO VIDAL
}

\begin{abstract}
We work in the smooth category: manifolds and maps are meant to be smooth. Let $G$ be a finite group acting on a connected closed manifold $X$ and $f$ an equivariant self-map on $X$ with $f_{\mid A}$ fixpointfree, where $A$ is a closed invariant submanifold of $X$ with codim $A \geq 3$. The purpose of this paper is to give a proof using obstruction theory of the following fact: If $X$ is simply connected and the action of $G$ on $X-A$ is free, then $f$ is equivariantly deformable rel. A to fxed point free map if and only if the usual Lefschetz number $L\left(f_{\mid(X, A)}\right)=0$. As a consequence we obtain a special case of a theorem of Wilczynski ( cf. [12, Theorem A]).

Finally, motivated by Wilczynski's paper we present an interesting question concerning the equivariant version of the converse of the Lefschetz fixed point theorem.
\end{abstract}

\section{Preliminaries}

We recall here how the classical abstruction theory of deformations of mappings (cf. [1]) can be translated to the equivariant case (cf. [3]).

Let $G$ be a finite group, $(Y, B)$ a pair of $G$-spaces and $(K, L)$ a given pair of finite regular $G$-complexes (cf. $[4$, p. 116] for the definition). A $G$-map $h:(K, L) \longrightarrow(Y, B)$ is said to be equivariantly deformable into $B$ if there exists a $G$-homotopy $h_{t}:(K, L) \longrightarrow(Y, B), t \in I=[0,1]$, such that $h_{o}=h$ and $h_{1}(K) \subset B$. We say that $h$ is equivariantly $q$-deformable into $B$, if the partial map $h_{\left(\bar{K}^{q}, L\right)}$ is equivariantly deformable into $B, \bar{K}^{q}:=K^{q} \cup L, K^{q}=q-$ skeleton of $K$. Note that for regular $G$-complexes $K$ we have that if $g \in G$ leaves any point $x \in X$ fixed, then $g$ must leave the smallest subcomplex of $K$ containing $I$ pointwise fixed. Therefore, by an easy inductive argument on the skeletons of $K$ we can show that $K$ has the equivariant extension property with respect to $L$. This allows us to assume that $h_{t}(x)=x$ for every $x \in L$ and $t \in I$ in the definition of deformability.

Let us assume that $B^{H} \neq \emptyset$ for all the subgroups $H$ of $G$ that occur as isotropy groups of simplexes in $K$, and both $B^{H}$ and $Y^{H}$ are pathwise connected. Then it is clear that $h$ must be equivariantly $O$-deformable into $B$.

The author would like to thank Professors Fadell and Kwasik for helpful suggestions 
Moreover, if the inclusion $B^{H} \subset Y^{H}$ induces an epimorphism of fundamental groups $\pi_{1}\left(B^{H}, y\right) \longrightarrow \pi_{1}\left(Y^{H}, y\right)$ for all $y \in B$, then it can be proved easily, as in the non equivariant case, that every $\operatorname{map} h$ is equivariantly 1 -deformable into $B$.

Next, let $q \geq 2$ and assume that $\left(Y^{H}, B^{H}\right)$ is $q$-simple for all subgroups $H$ of $G$. Then the relative homotopy groups $\pi_{q}\left(Y^{H}, B^{H}\right)$ are abelian and can be used to define the following generic coefficient system for $G, \tilde{\omega}_{q}(G / H):=$ $\pi_{q}\left(Y^{H}, B^{H}\right)(\mathrm{cf} .[3])$. Now suppose that $h\left(\bar{K}^{q-1}\right) \subset B$. For each orientable $q$ simplex $\sigma$ in $K-L$ define an element $d_{G}^{q}(h)(\sigma) \in \pi_{q}\left(Y^{G}, B^{G}{ }^{\circ}\right), G_{\sigma}$ the isotropy group of $\sigma$, to be the element determined by the map $h:(\sigma, \dot{\sigma}) \longrightarrow\left(Y^{G \sigma}, B^{G \sigma}\right)$. Since this last pair is simple it does not matter how the base points are choosen in the definition of $d_{G}^{q}(h)$. We can prove that $d_{G}^{q}(h)$ is a relative equivariant coclycle of $K$ modulo $L$ and $d_{G}^{q}(h)=0$ if and only if $h$ sel. $\bar{K}^{q-1}$ equivariantly $q$-deformable into $B$.

Now consider the $n$-dimensional obstruction set $0_{G}^{q}(h)$ to the equivariant deformation of the map $h$. It is a subset of the Bredon cohomology group $H_{G}^{q}\left(K, L ; \tilde{\omega}_{Q}\right)$ (cf. $[3 \mid$ ) and is defined as in the classical case. We have that $0_{G}^{q}(h) \neq \emptyset$ if $h$ is equivariantly $q-1$ deformable into $B$ and if $h$ is equivariantly $q$-deformable into $B$, then $0_{G}^{q}(h)$ contains the zero element. Next we wish to indicate how the converse of this latter statement can be proved.

Let $h$ be equivariantly $q-1$ deformable into $B, h_{0}, h_{1}:(K, L) \longrightarrow(Y, B)$ two equivariant maps which satisfy the conditions $h_{\mathbf{i}}\left(\overline{K^{Q-1}}\right) \subset B, h_{\mathbf{i}} \underset{G}{\simeq} h, i=0,1$, and $h_{0 \mid \bar{K}^{q-\gamma}}=h_{I \mid \bar{K}^{q-z}}$. Then it is possible to associate to any equivariant homotopy $h_{t}$ between $h_{0}$ and $h_{\mathrm{I}}$ which has the property that $h_{t}(x)=h_{0}(x)=$ $h_{1}(x)$ for all $x \in \bar{K}^{q-2}$ an equivariant separation cochain $d_{G}^{q-1}\left(h_{t}\right) \in\left(K, L ; \tilde{\omega}_{q}\right)$ satisfying the equation

$$
\delta d_{G}^{q-1}\left(h_{t}\right)=d_{G}^{q}\left(h_{1}\right)-d_{G}^{q}\left(h_{0}\right)
$$

This separation cochain is defined as follows: Let $h_{t}^{H}$ be the restriction of $h_{t}$ to $K^{H}$. By the classical theory applied to this map (cf. [1]), there exists a cochain $d^{q-1}\left(h_{t}^{H}\right) \in C^{q-1}\left(K^{H}, L^{H} ; \bar{\omega}_{q}(G / H)\right)$ with $\delta d^{q-1}\left(h_{t}^{H}\right)=d^{q}\left(h_{1}^{H}\right)-d^{q}\left(h_{0}^{H}\right)$. Because we are dealing with equivariant maps we have $\tilde{\omega}_{q} d^{q-1}\left(h_{t}^{H^{\prime}}\right)(\sigma)=$ $d^{g-1}\left(h_{t}^{H}(g \sigma)\right)$ for $g: G / H \rightarrow G / H^{\prime}$ a morphism in the orbit category and

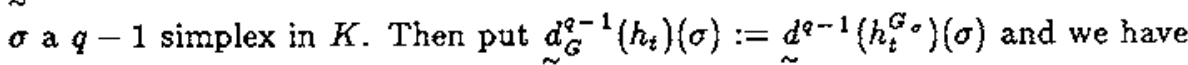
${\underset{\sim}{G}}_{G}^{q-1}\left(h_{t}\right) \in C_{G}^{q-1}\left(K, L ; \tilde{\omega}_{q}\right)$ satisfying the equation (1).

Furthermore, if $d$ is an element of the cochain group $C_{G}^{q-1}\left(K, L ; \bar{\omega}_{q}\right)$ and $h$ is any map satisfying the condition $h\left(\bar{K}^{q-1}\right) \subset B$, then it can be defined an equivariant deformation $h_{t}$ of $h$ such that $h_{t}(x)=h(x)$ for all $x \in \bar{K}^{q-2}, h_{1}\left(\bar{K}^{q-1}\right) \subset$ $B$ and $d_{G}^{q-1}\left(h_{t}\right)=d$ (cf. $[3]$ ). Finally, by making use of this separation cochain we can easily prove the following: 
1.1. Proposition. The $G$-map $h$ is equivariantly $q$-deformable into $B$ if and only if $o \in 0_{G}^{q}(h)$.

Suppose now that $(Y, B)$ is a $G$-locally trivial pair over $K$; that means we have a $G$-map $p: Y \rightarrow K$ with $p(B)=K$ and for each $x \in K$ there is a $G_{x}$-invariant neighborhood $U$ of $x$ and $G_{z}$-homeomorphism of pairs

$$
\Psi_{U}:\left(p^{-1} U, p_{o}^{-1} U\right) \longrightarrow\left(U \times p^{-1}(x), U \times p_{o}^{-1}(x)\right)
$$

such that $p \Psi_{U}(x, y)=x$ for all $(x, y) \in U \times p^{-1}(x)$ and $p_{o}:=p_{\mid B}$.

It is known that a $G$-locally pair over a $G$-complex is an equivariant (Serre) fibration (cf. [2]). Therefore we have the equivariant version of Lemma $5.1 \mathrm{in}$ $[5]$ and consequently the following

1.2. Proposition. If $h$ is a cross section of the $G$-locally trivial pair $(Y, B)$ over $K$, and $h$ is equivariantly deformable into $B$ then it is also in the family of cross sections.

\section{Application to selfmaps on manifolds}

Throughout we assume maps and manifolds are smooth. Let $G$ be a fnite group acting on a pathwise and simply connected closed manifold $X$. Let $f:(X, A) \rightarrow(X, A)$ be a $G$-map where $A$ is closed invariant submanifold of $X$ and $f_{\mid A}$ is fxed point free. In order to apply the preceeding material to this situation let us assume that the manifolds $X^{H}$ are pathwise and simple connected for all subgroups $H$ of $G$. Choose an equivariant triangulation of $X$ with $A$ a $G$-invariant subcomplex (cf. [7, p. 216]). Considered locally at $x \in X$, the $G$-manifold $X$ looks like a $G_{z}$-representation in the tangent space $T_{z} X$ (cf. [8]). Consequently, if $\Delta$ is the diagonal, it can easily be proved that the pair $\hat{X}:=(X \times X, X \times X-\Delta)$ with $p: X \times X \rightarrow X$ the projection on the first factor is a $G$-locally trivial pair over the $G$-complex $X$. By Proposition 1.2 the $G$-map $f$ is equivariantly deformable to a fixed point free map if and only if the cross section $(1, f): X \rightarrow X \times X,(1, f)(x):=(x, f(x))$, is equivariantly deformable into $X \times X-\Delta$. Finally, by repeated application of Proposition 1.1 for $(K, L):=(X, A),(Y, B):=(X \times X, X \times X-\Delta)$ and $h:=(1 ; f)$, we obtain the following result:

2.1. Theorem. The $G$-map $f:(X, A) \rightarrow(X, A)$, with $f_{\mid A}$ fixpointfree, is equivariantly deformable rel. A to fixed point free map if and only if $o \in$ $0_{G}^{n}(f) \subset H_{G}^{n}\left(X, A ; \tilde{\omega}_{n}\right)$, where $n=\operatorname{dim} X$ and $\tilde{\omega}_{n}$ is the coefficient system given by $\tilde{\omega}_{n}(G / H):=\pi_{n}\left(\hat{X}^{H}\right)$.

To simplify the notation we have written $0_{G}^{n}(f)$ instead of $0_{G}^{n}((1, f))$. Note that $o \in 0_{G}^{n}(f)$ if and only if $a \in 0_{G}^{q}(f)$ for all $q, 2 \leq q \leq n$. For a given 
equivariant triangulation of $(X, A)$, only the $q$-simplexes $\sigma$ in $X-A$ with $q=\operatorname{dim} X^{G} \sigma$ contribute in a non trivial manner to the obstruction set $0_{G}^{q}(f)$. This is because for $q<\operatorname{dim} X^{G_{\sigma}}$ we have $\pi_{q}\left(\hat{X}^{G_{\sigma}}\right)=0$. Finally there is the fact that the condition $o \in 0_{G}^{n}(f)$ does not depend on the triangulation we have choosen. This follows from the Theorem 2 in [7] together with the observation that after equivariant subdivision, the inclusion of the given triangulation into a subdivision, induces an isomorphism in cohomology, mapping in a 1-1 fashion one obstruction set into the other.

In order to relate the condition $o \in 0_{G}^{n}(f)$ of Theorem 2.I with the Lefschetz number of the map $f$ we assume that $G$ acts freely on $X-A$ and $\operatorname{codim} A \geq 3$. Our next step is to prove that in this special case the group $H_{G}^{n}\left(X, A ; \tilde{\omega}_{n}\right)$ is torsion free.

Let $U$ be a closed invariant tubular neighborhood of $A$ equivariantly diffeomorphic to an $\varepsilon>0$ disc bundle $D_{e}$ in the normal bundle $N(A, X)$ to $A$ in $X$, via $h: U \rightarrow D$ (cf. $[4$, p.306]). Then $A$ is an equivariant strong deformation retract of $U$, and the groups $H_{G}^{n}\left(X, A ; \tilde{\omega}_{n}\right)$ and $H_{G}^{n}\left(X, U ; \tilde{\omega}_{n}\right)$ are isomorphic. Let $V:=h^{-1}\left(D_{e / 2}\right)$. Clearly $V \subset \stackrel{\circ}{U}$, and the inclusion $(X-\stackrel{\circ}{V}, U-\stackrel{\circ}{V}) \subset(X, U)$ induces an isomorphism $H_{G}^{n}\left(X, U ; \bar{\omega}_{n}\right) \simeq H_{G}^{n}\left(X-\stackrel{\circ}{V}, U-\stackrel{\circ}{V} ; \bar{\omega}_{n}\right)$.

Consider now the following equivariant deformation $\emptyset_{t}: X-A+X-A$ of the identity on $X-A$. For $x \in X-A$ we define

$$
\emptyset_{i}(x):= \begin{cases}x & \text { if } \quad x \in X-\stackrel{O}{ } \\ h^{-1} \rho_{t} h(x) & \text { if } \quad x \in U\end{cases}
$$

where $\rho_{t}: D_{\varepsilon}-N_{0} \longrightarrow D_{\varepsilon}-N_{0}$ and $N_{0}=$ the zero cross section is defined by $\rho_{t}(x, v):=(x,(1-t) v+t \varepsilon v /\|v\|)$. On $N(A, X)$ we have an ortogonal metric, hence the maps $\rho_{t}$ are $G$-maps and, consequently, the maps $\emptyset_{t}$ are equivariant diffeomorphisms. However $\emptyset_{1}(X-\stackrel{\circ}{V})=X-\stackrel{\circ}{U}$ and $\emptyset_{1}(U-\stackrel{\circ}{V})=U-\stackrel{\circ}{U}$, hence $\emptyset_{1}$ induces an isomorphism $\emptyset_{1}^{*}: H_{G}^{n}\left(X-\stackrel{\circ}{V}, U-\stackrel{\circ}{V} ; \dot{\omega}_{n}\right) \longrightarrow H_{G}^{n}\left(X-\stackrel{\circ}{U}, U-\stackrel{\circ}{U} ; \bar{\omega}_{n}\right)$, where $(X-\stackrel{\circ}{U}, U-\stackrel{\circ}{U})$ is an $n$-dimensional compact manifold with boundary on which $G$ acts freely. Furthermore if the $\operatorname{codim} A \geq 3$, then we have that path components of $X-A$ are simply connected, and consequently the manifold $X-\stackrel{\circ}{U}$ is also simply connected. Finally, put $(Y, \partial Y):=(X-\stackrel{\circ}{U}, U-\stackrel{\circ}{U})$.

2.2. Lemma. With the previous notation and taking the orientation induced on $Y$ by that on $X$, we have that the group $H_{G}^{n}\left(Y, \partial Y ; \tilde{\omega}_{n}\right)$ is torsion free.

Proof: First we note that the group $H_{G}^{n}\left(Y, \partial Y ; \bar{\omega}_{n}\right)$ is, by definition, equal to the group $H^{n}\left(Y / G, \partial Y / G ; \pi_{n}(\hat{X})\right)$ where $\pi_{n}(\hat{X}) \cong \mathbb{Z}$ have the right $Z G$-module structure given by the coefficient system $\tilde{\omega}_{n}$. Second, and this is essential for 
the proof, we use the fact that if we consider the orientation on. $Y$ induced by that on $X$, then the $G$-module structure on $\mathbf{Z}$ coincides with the orientation homomorphism $\omega: \pi_{1}(Y / G) \longrightarrow \mathbb{Z}_{2}$ for the manifold $Y / G$, that is $\tilde{\omega}_{n}(g)=I_{\mathbb{Z}}$ if $\omega(g)=1$ and $\tilde{\omega}_{n}(g)=-1_{z}$ if $\omega(g)=-1$.

By Theorem 2.1 in [11], $(Y, \partial Y)$ is a Poincaré pair of formal dimension $n$. Then Lemma 1.2 in [10] yields that the cup product with the fundamental class

$$
[Y / G, \partial Y / G] \cap: H^{n}(Y / G, \partial Y / G ; Z) \longrightarrow H_{\circ}\left(Y / G ; \mathbb{Z}^{t}\right)
$$

is an isomorphism, where $\mathbb{Z}$ is considered with the $\mathbb{Z} G$-right module structure given by $\tilde{\omega}_{n}$ and $\mathbb{Z}^{i}$ is the left $\mathbb{Z} G$-module defined as follows. For $\lambda=\operatorname{Ln}(g) g \in$ Z. $G$ let $\bar{\lambda}:=\Sigma n(g) \omega(g) g^{-1}$ and define $g z:=z \bar{g}=\omega(g) \tilde{\omega}_{n}(g)(z)$. However with the above choice $\omega(g) \bar{\omega}_{n}(g)$ is always the identity and $Z^{t}$ is trivial as $\mathbb{Z} G$-left module. Therefore $H_{\diamond}\left(Y / G ; \mathbb{Z}^{t}\right)$ is a free abelain group with as many generators as there are path components in $Y / G$. This proves the lemma.

Now consider the homomorphism induced in cohomology by the inclusion $C_{G}^{*}\left(X, A ; \bar{\omega}_{n}\right) \longrightarrow C^{*}(X, A ; \mathbb{Z})$,

$$
\alpha: H_{G}^{*}\left(X, A ; \tilde{\omega}_{n}\right) \longleftrightarrow H^{*}(X, A ; \mathbb{Z}): \beta .
$$

In the opposite direction, let $\beta$ be the transfer. At the cochain level, it is defined as follows: $\beta z(\sigma):=\sum_{g \in G} \tilde{\omega}_{n}(g) z\left(g^{-1} \sigma\right), z \in Z^{n}\left(X, A ; \pi_{n}(\hat{X})\right)$. We have that the composition $\beta \alpha=-|G|$, multiplication with $|G|$, the order of the group $G$. From this equation and the fact that the group $H_{G}^{n}\left(X, A ; \tilde{\omega}_{n}\right)$ has no torsion, it follows that $\alpha$ must be monomorphism.

On the other hand $\alpha$ maps the $n$-dimensional obstruction set $0_{G}^{n}(f)$ into the classical one. This last set has been computed by Fadell in [5]. It has only one element, the Lefschetz class of $f_{\mid(X, A)}$ with coefficients in $\mathbb{Z}$, and this is zero if and only if the usual Lefschetz number $L\left(f_{\mid(X, A)}\right)=0$. In summary we have the following:

2.3. Theorem. Assuming in addition to the hypothesis of Theorem 2.1 that $G$ acts freely on $X-A$ and codim $A \geq 3$, we have that the $G$-map $f:(X, A) \longrightarrow$ $(X, A)$ is equivariantly deformable rel. $A$ to a fixed point free map if and only if $L(f(x, A))=0$.

2.4. Corollary. Suppose $G$ acts semifreely on $X$ with $X^{G}$ a pathwise and simply connected manifold of codim $X^{G} \geq 3$. Then a $G$-map $f: X \rightarrow X$ is equivariantly deformable to a fixed point free map if and only if the Lefschetz numbers $L(f)=L\left(f^{G}\right)=0$.

2.5. Corollary. Suppose $G$ acts freely on $X$. Then a $G$-map $f: X \rightarrow X$ is equivariantly deformable to a fixed point free map if and only if the Lefschetz number $L(f)=0$.

This last corollary in the case $\operatorname{dim} X=2$ is particulary simple. Here $X$ must be equivariantly diffeomorphic to $S^{2}$, with the antipodal action of $\mathbb{Z}_{2}$. An 
equivariant map $f$ with $L(f)=0$ has degree -1 and is equivariantly homotopic to the antipodal map (cf. [9, p. 212]).

We can also mention here the corollary 6.36 in [6]. There Fadell gives, in a particular case, an obstruction theory proof of Corollary 2.5. He assumes in addition that all maps $g: X \longrightarrow X$ are homotopic and proves that the induced map $f / G: X / G \longrightarrow X / G$ can be deformed to a fixed point free map. Consequently, the map $f$ is equivariantly homotopic to a fixed point free map. We have also proved this last fact, as in the case of the antipodal action of $\mathbb{Z}_{2}$ on $S^{2 n}$, where all induced maps on $R P^{2 n}$ have fixpoints.

2.6. Remark. The condition codim $X^{G} \geq 3$ in the Corollary 2.4 is only necessary in our proof of Theorem 2.3 (see comments before Lemma 2.2). However, our Corollary also holds without restrictions on the codimension. To see this, it is enough to note that the space $X-X^{G} / G$ of orbits is connected and consequently the Wilczynski invariant (cf. $[12, \mathrm{p} .50]$ ) vanishes if we assume that the Lefschetz numbers $L\left(f^{G}\right)$ and $L(f)$ are zero.

We denote by $G$-simply connected manifold a $G$-manifold $X$ such that $X^{H}$ is 0 and 1-connected for all subgroups $H$ of $G$.

Problem. Let $f: X \rightarrow X$ be a G-map, where $X$ is a $G$-simply connected manifold. Suppose that the equivariant fixed point index of $f$ is zero or equivalenty, $L\left(f^{H}\right)=0$ for all stubgroups $H$ of $G$. It is then true that if $f$ is equivariantly deformable to a fixed point free map?

Notice that what we need to prove is that the Wilczynski invariant of $f$ is zero. For free and semifree actions the answer of this question is yes. But I do not know the answer in the general case. A negative answer would provide an example of an equivariant property of a $G$-map $f$, i.e. "equivariant deformability to a fixed point free map ", which does not necessary hold even if the maps $f^{H}: X^{H} \longrightarrow X^{H}$ are deformable to fixed point free maps in the classica! sense.

\section{References}

1. BLAKERS A.L.; MASSEY W.S., The homotopy group of a triad I., Annals of Math. 53 (1951).

2. BIERSTONE E., The equivariant covering homotopy property for differentiable G fibre bundles, J. of Diff. Geom. 8 (1973).

3. BREDON G., Equivariant cohomologies theories, Lecture notes in Math 34 (1967), Springer.

4. BREDON G., Introduction to compact transformation groups, Academic Press (1972).

5. FADELL E., On the coincidence theorem of F.B. Fuller, Pac. J. of Math. 15 (1965). 
6. FADELL E.; HUSSEINI S., Fixed point theory for non simply connected manifolds, Topology 20 (1981).

7. ILLMAN S., Smooth equivariant triangulations of $G$-manifolds for $G$ a finite group, Math. Annalen 233 (1978).

8. JäNICH K., Differenzierbare G-Mannigfaltigkeiten, Lecture Notes in Math. 59, Springer.

9. TOM DIECK T., Transformation groups and representation theory, Lecture Notes in Math 766 (1979), Springer.

10. Wall C.T.C., On Poincaré complexes I, Annals of Math 86 (1967).

11. Wall C.T.C., Surgery on compact manifolds, Academic Press (1970).

12. WILCZYNSKI D., Fixed point free equivariant homotopy classes, Fund. Math. 123 (1984).

Institut d'Estudis Catalans

Centre de Recerca Matematica

Apartat 50, Bellaterra (Barcelona), SPAIN.

current address: Universidad de La Laguna

Facultad de Matemáticas

La Laguna, Tenerife, SPAIN.

Rebut el 16 de Juny de 1987 